\title{
The tryptophan derivative, tranilast, and conditioned medium with indoleamine 2,3-dioxygenase-expressing cells inhibit the proliferation of lymphoid malignancies
}

\author{
SHIHOKO SUWA*, AYA KASUBATA*, MIYU KATO, MEGUMI IIDA, \\ KEN WATANABE, OSAMU MIURA and TETSUYA FUKUDA \\ Department of Hematology, Graduate School of Medical and Dental Sciences, \\ Tokyo Medical and Dental University, Bunkyo-ku, Tokyo 113-8519, Japan
}

Received October 9, 2014; Accepted November 25, 2014

DOI: $10.3892 /$ ijo.2015.2825

\begin{abstract}
Indoleamine 2,3-dioxygenase (IDO) is an enzyme that catalyzes tryptophan degradation and induces immunosuppression. Although IDO is an important factor that allows tumors to escape from immunological attack, its effect on lymphoid malignancies has not been fully revealed. We evaluated the expression of $I D O$ in samples from patients with B-cell malignancies. The IDO expression in the tumor samples was comparable to those in peripheral blood mononuclear cells from healthy donors and had mainly originated from non-B cell populations. We introduced IDO gene into Chinese hamster ovary $(\mathrm{CHO})$ cells. We then cultured various cell lines using $\mathrm{CHO}$ - or CHO-IDO-conditioned medium. Compared with the $\mathrm{CHO}$ medium (CHO-CM), the CHO-IDO medium (IDO-CM) decreased the viability of lymphoid cell lines but not those of the non-lymphoid lines. Next, we examined the effects of tryptophan metabolites on lymphoid tumors, and revealed that the drug N-[3',4'-dimethoxycinnamoyl] anthranilic acid (tranilast), a synthetic derivative of the tryptophan metabolite, was able to repress proliferation and dose-dependently induce cell death of lymphoid cell lines. Tranilast induced the activation of the c-Jun N-terminal kinase, which is activated by cellular stress, in lymphoid cells. The effect of tranilast on lymphoid cells was independent of the aryl hydrocarbon receptor (AhR) although tranilast has been reported to be an AhR agonist. Finally, the administration of tranilast decreased murine lymphoid tumor progression in vivo. These results indicated that IDO and tryptophan derivatives, particularly tranilast, can be tools for the therapy for lymphoid malignancies.
\end{abstract}

Correspondence to: Dr Tetsuya Fukuda, Department of Hematology, Graduate School of Medical and Dental Sciences, Tokyo Medical and Dental University, Yushima 1-5-45, Bunkyo-ku, Tokyo 113-8519, Japan E-mail: fuku.hema@tmd.ac.jp

*Contributed equally

Key words: indoleamine 2,3-dioxygenase, tryptophan, tranilast, lymphoid malignancy

\section{Introduction}

Indoleamine 2,3-dioxygenase (IDO) is an enzyme that catalyzes the essential amino acid tryptophan and leads to the starvation of tryptophan and the accumulation of tryptophan metabolites such as kynurenine. Therefore, it induces apoptosis or cell cycle arrest of activated $T$ cells and the differentiation of new regulatory $\mathrm{T}$ cells, which results in immunologic suppression (1-3). This control may be essential for physiological acquired immunological tolerance. For example, IDO expressed by the placenta prevents a maternal allo-reaction against the fetus $(4,5)$ and enables the maintenance of gestation as shown by the fact that the IDO inhibitor, 1-methyl-tryptophan (1-MT), induced the immune-mediated rejection of allogeneic concepti (6). IDO is also expressed in some tumor cells, and it plays a pivotal role in the escape from immunological attack by inducing immunological anergy in such tumors $(7,8)$. 1-MT could potentiate antitumor immunity in these IDO-expressing tumors, and could induce tumor regression in animal models (9). Based on this result, several clinical trials evaluating the use of 1-MT against solid tumors are currently ongoing. However, the role of IDO in lymphoid tumors such as lymphoma or lymphocytic leukemia has not been fully revealed.

Experimental autoimmune encephalomyelitis (EAE) is a well-known animal model of multiple sclerosis and is mediated by autoimmune $\mathrm{T}_{\mathrm{H}} 1$ cells (10). Altered peptide ligands (APLs) that had modified the strength of T-cell receptor signaling had induced self-tolerance to autoantigens. Platten et al (10) showed that the IDO expression was induced by APLs and that the administration of $\mathrm{N}$-[3',4'-dimethoxycinnamoyl] anthranilic acid (tranilast), a synthetic derivative of the tryptophan metabolite anthranilic acid, could reverse paralysis in mice with EAE. IDO has also been reported to be associated with acute graft-versus-host disease (GVHD) in murine bone marrow transplantation. IDO expression is induced at the site of GVHD. Moreover, exogenous kynurenines can reduce GVHD lethality $(11,12)$. These reports indicated that the tryptophan metabolites as well as the forced IDO expression may have some effect on the fate of activated lymphocytes.

It is possible that an analysis of the expression and effect of IDO in lymphoid malignancies will provide a clue 
regarding their treatment. In the present study, we found that the lymphoid malignant cells were susceptible to cytotoxicity by IDO and that tranilast also induced growth suppression of these cells.

\section{Materials and methods}

Ethics statement. After written informed consent was obtained in compliance with the Declaration of Helsinki, samples (peripheral blood, bone marrow, lymph node, ascites and pleural effusion) were collected from the patients. Approval was obtained from the ethics committee of the Tokyo Medical and Dental University. All animal studies were approved by the Animal Subjects Committee of the Tokyo Medical and Dental University and were performed in accordance with the institutional guidelines.

Cells and reagents. Splenocytes were obtained from 8-week-old female BALB/c mice using a RBC lysis buffer $\left[0.155 \mathrm{M} \mathrm{NH}_{4} \mathrm{Cl}\right.$, $0.01 \mathrm{M} \mathrm{NH}_{4} \mathrm{HCO}_{3}$ and $0.1 \mathrm{mM}$ EDTA (pH 7.5)]. Peripheral blood mononuclear cells (PBMCs) from patients were isolated through density-gradient centrifugation from freshly collected blood samples using SEPARATE-L (Muto Pure Chemicals, Tokyo, Japan). Cells were preserved with CELLBANKER (Nippon Zenyaku Kogyo Co., Ltd., Fukushima, Japan) at $-80^{\circ} \mathrm{C}$. Human and murine cell lines were obtained from the American Type Culture Collection (ATCC, Manassas, VA, USA) and cultured in RPMI-1640 medium or Dulbecco's modified Eagle's medium (DMEM) medium with $10 \%$ fetal calf serum (FCS). Tranilast was provided by Kissei Pharmaceutical (Nagano, Japan). IFN $\gamma$, concanavalin A (ConA), L-kynurenine, 3-HAA, Kynurenic acid, fludarabine, $\alpha$-naphthoflavone, and 2,3,7,8-Tetrachlorodibenzodioxin (TCDD) were purchased from Sigma-Aldrich (St. Louis, MO, USA). IL-2 was purchased from R\&D Systems (Minneapolis, MN, USA). CD19 Pan B Dynabeads and human T-activated CD3/CD28 Dynabeads were purchased from Invitrogen Life Technologies (Grand Island, NY, USA). 4-Hydroperoxy Cyclophosphamide were purchased from Wako Pure Chemical Industries (Osaka, Japan). JNK inhibitor SP600125 was purchased from Enzo Life Sciences (Farmingdale, NY, USA).

Real time $(R T)-P C R$. Samples from patients with B-cell lymphoma were used. RNA was isolated using TRIzol (Gibco-BRL, Gaithersburg, MD, USA). Reverse transcription using an oligo dT primer was performed with a SuperScript II RT kit (Invitrogen-Life Technologies, Grand Island, NY, USA). The synthesized cDNA was amplified with primers specific for human IDO (forward, 5'-CCTGACTTATGAGAA CATGGACGT-3' and reverse, 5'-ATACACCAGACCGTCTG ATAGCTG-3'); or mouse IDO (forward, 5'-TTCGAAAGGTG CTGCCCCGC-3' and reverse, 5'-GCCCTTGTCGCAGTCCC CAC-3'); or human GAPDH (forward, 5'-CTGACTTCAAC AGCGACACC-3' and reverse, 5'-TCCTCTTGTGCTCTTGC TGG-3'); or mouse GAPDH (forward, 5'-TGCGACTTCAAC AGCAACTC-3' and reverse, 5'-CTTGCTCAGTGTCCTTGC TG-3'). Quantitative RT-PCR was performed with the LightCycler FastStart DNA Master Plus SYBR-Green I kit using LightCycler software version 3.5 (Roche Applied Science, Indianapolis, IN, USA).
Cloning of mouse IDO (mIDO). The murine Ido-1 cDNA containing the full length open reading frame was amplified with the specific primers (forward, 5'-GGAGTAGA CAGCAATGGCAC-3' and reverse, 5'-GAGCTTGCTA CACTAAGGCC-3') using the RNA from splenocytes as a template. The purified product of $1250 \mathrm{bp}$ was cloned into the pGEM-T Easy Vector system (Invitrogen-Life Technologies). After sequencing, it was subcloned into the expression vector, pcDNA3 (Invitrogen-Life Technologies) (pcDNA3-mIDO).

Transfection. Chinese hamster ovary (CHO) cells were transfected with pcDNA3-mIDO using Lipofectamine LTX (Invitrogen-Life Technologies) and selected with $1 \mathrm{mg} / \mathrm{ml}$ geneticin (G418) (Roche Applied Science). The stable transfected $\mathrm{CHO}$ cells were subcloned, and the clones with high levels of mIDO protein expression were selected. Furthermore, pcDNA3-mIDO was transfected to the murine B lymphoma cell lines A20 and M12 by electroporation. RNA and protein were extracted from the transfected cells after selection with G418. A plasmid containing the dominant negative form of c-Jun N-terminal kinase (JNK) was obtained from the Addgene repository [13761; pcDNA3 Flag Jnk2a2 (apf)] (13). The coding region (DN-JNK2) was subcloned into retroviral vector pQCXIN (Clontech: Palo Alto, CA, USA). A20 cells were transduced with pQCXIN-DN-JNK2 and selected with G418.

Conditioned medium. We cultured $\mathrm{CHO}$ cells transfected with the $m I D O$ gene or control vector in a minimum essential medium ( $\alpha$ MEM) medium. On the following day, the $\alpha$ MEM in the dish was changed to RPMI-1640 medium and the $\mathrm{CHO}$ cells were cultured for 5 days at $37^{\circ} \mathrm{C}$ and in $5 \% \mathrm{CO}_{2}$. The collected supernatant from the dish was adjusted to a $\mathrm{pH}$ of 7.0 with $1 \mathrm{~N} \mathrm{NaOH}$ and then $2 \mathrm{mM}$ L-glutamine (Invitrogen-Life Technologies) was added. This medium was sterilized by a filter.

Measurement of cell viability and viable cell number. In the present study, the determination of cell viability was based on an analysis of mitochondrial transmembrane potential using 3,3'-dehexyloxacarbocyamine iodine (DiOC6) (Molecular Probes, Eugene, OR, USA) and the cell membrane permeability to propidium iodide (PI) (Molecular Probes) by flow cytometry with a FACSCalibur ${ }^{\mathrm{TM}}$ flow cytometer (Becton-Dickinson, Franklin Lakes, NJ, USA) as previously described (14). The fluorescence-activated cell sorting (FACS) data were analyzed with FlowJo software version 6.3.3 (Tree Star, Inc., Ashland, OR, USA). The DiOC6-positive and PI-negative cells were considered to be viable cells. The viable cell number was calculated as the cell number that was acquired during $30 \mathrm{sec}$ multiplied by the percentage of viable cells.

Immunoblot analysis. Cells were lysed in a lysis buffer containing $1 \%$ Triton $\mathrm{X}-100,20 \mathrm{mM}$ Tris- $\mathrm{HCl}(\mathrm{pH} 7.5), 150 \mathrm{mM}$ $\mathrm{NaCl}, 1 \mathrm{mM}$ EDTA, $1 \mathrm{mM}$ sodium orthovanadate, $1 \mathrm{mM}$ phenylmethylsulfonyl fluoride, and $10 \mathrm{mg} / \mathrm{ml}$ each of aprotinin and leupeptin (Sigma-Aldrich, St. Louis, MO, USA). The cell lysates were separated using sodium dodecyl sulfate-polyacrylamide gel electrophoresis (SDS-PAGE), and electroblotted 

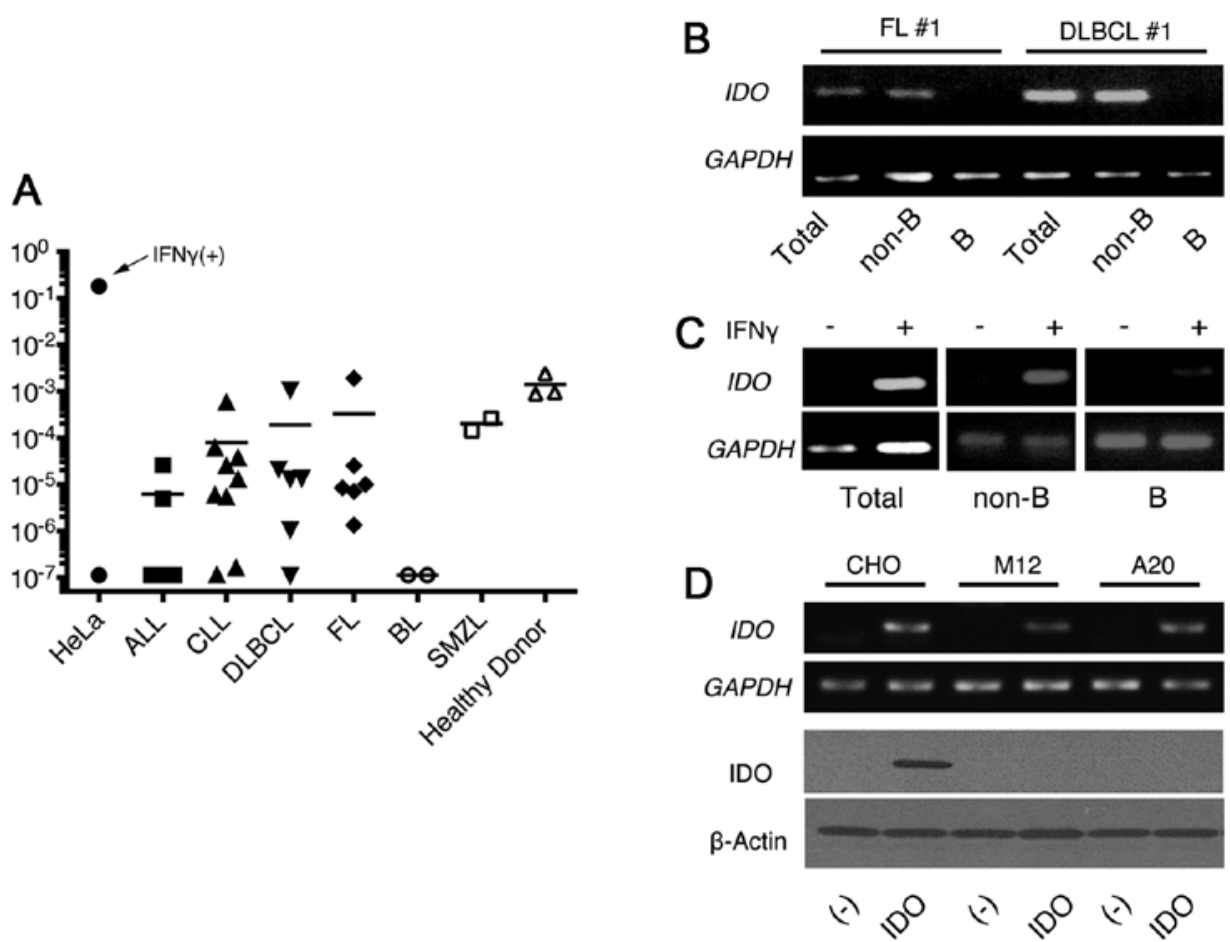

Figure 1. Indoleamine 2,3-dioxygenase (IDO) expression in B-lymphoid malignancies. (A) IDO expression in 30 clinical samples from B-lymphoid malignancies [acute lymphocytic leukemia (ALL) n=5; chronic lymphocytic leukemia (CLL) n=9; diffuse large B-cell lymphoma (DLBCL) $n=6$; follicular lymphoma (FL) n=6; Burkitt's lymphoma (BL) n=2; splenic marginal zone lymphoma (SMZL) n=2], 3 samples from healthy donors, positive controls (HeLa cells treated with $10 \mathrm{ng} / \mathrm{ml}$ IFN $\gamma$ ), and negative controls (untreated HeLa cells) were analyzed using quantitative real-time PCR (Rq-PCR) and showed the ratio IDO to GAPDH (internal control). Each symbol represents an individual patient. (B) IDO expression was analyzed in the total cells, B cells and non-B cells from the clinical samples of the patients with FL and DLBCL after the separation with CD19 Pan B Dynabeads. (C) B and non-B cells were separated from peripheral blood mononuclear cells (PBMCs) from patients with B-CLL. IDO expression was analyzed after the addition of IFN $\gamma$ for 1 day. Five samples of different patients were analyzed, and representative results are shown. (D) IDO mRNA and protein expression in Chinese hamster ovary (CHO) cells, and in murine B-cell lines A20 and M12 in which mouse IDO or control pcDNA3 vector was transfected.

to polyvinylidene difluoride membranes. Membranes were probed with primary antibodies and probed with HRP that was conjugated anti-mouse or rabbit IgG (GE Healthcare Life Sciences, Piscataway, NJ, USA) as the second antibody. Antibodies against IDO (Oriental Yeast Co., Ltd., Tokyo, Japan), SAPK/JNK, phospho-Thr183/Tyr185-SAPK/JNK, AKT, phospho-S473-AKT, p38, phospho-Thr180/Tyr182-p38 (Cell Signaling Technology, Danvers, MA, USA) and $\beta$-actin (Sigma-Aldrich) were used.

Mice. Eight-week-old female BALB/c mice were purchased from CLEA Japan (Tokyo, Japan) and were housed under specific pathogen-free conditions. After intraperitoneal injection of anesthesia using 2,2,2-tribromoethanol (Avertin; Sigma-Aldrich), the bilateral hind flanks of each mouse were transplanted with $2 \times 10^{5}$ of $\mathrm{A} 20$, which was a B lymphoma cell line derived from the BALB/c mice. These cells generated palpable tumors at the site of injection in $100 \%$ of the injected mice. Seven days after the inoculation, tranilast that was suspended in $0.5 \%$ carboxymethyl cellulose (CMC) solution or CMC solution alone had been administered by oral gavages with a disposable flexible tube twice a day for 3 weeks. The tumor progression was monitored biweekly, and the tumor volumes were calculated with the following formula: Volume $=(\text { width })^{2} \mathrm{x}$ length $/ 2$.

Statistical analysis. The significance of the differences between groups was determined by the Mann-Whitney U test, the Student's t-test, or the Student's paired t-test using GraphPad Prism software (La Jolla, CA, USA).

\section{Results}

IDO expression in human B-lymphoid malignancies. First, we examined the $I D O$ expression in 30 samples from the patients with B-cell malignancies by Rq-PCR using specific primers for $I D O$ gene. We set HeLa cells that were treated with $10 \mathrm{ng} / \mathrm{ml}$ IFN $\gamma$ and untreated HeLa cells as the positive and negative controls, respectively. The expression of IDO from 22 samples was detected as 10-fold higher than those of the negative controls although all samples were less than one tenth of the positive controls. The results were comparable to the expression of PBMCs from three healthy donors. The expression was heterogeneous in the samples and had no clear correlation with the malignancy types (Fig. 1A) except for Burkitt's lymphoma (BL), which had no detectable expression. We further evaluated the IDO expression in the samples that had a relatively high expression after the separation of the B cells and non-B cells. The IDO expression was mainly detected in non-B cell populations in five examined samples (Fig. 1B). We then examined $I D O$ induction after IFN $\gamma$ treatment $(10 \mathrm{ng} / \mathrm{ml})$ using the PBMCs from CLL patients because this cytokine strongly induced IDO in dendritic cells. IDO was clearly upregulated after the treatment in PBMCs from patients with CLL. However, the findings demonstrated that this induction was associated with the non-B 

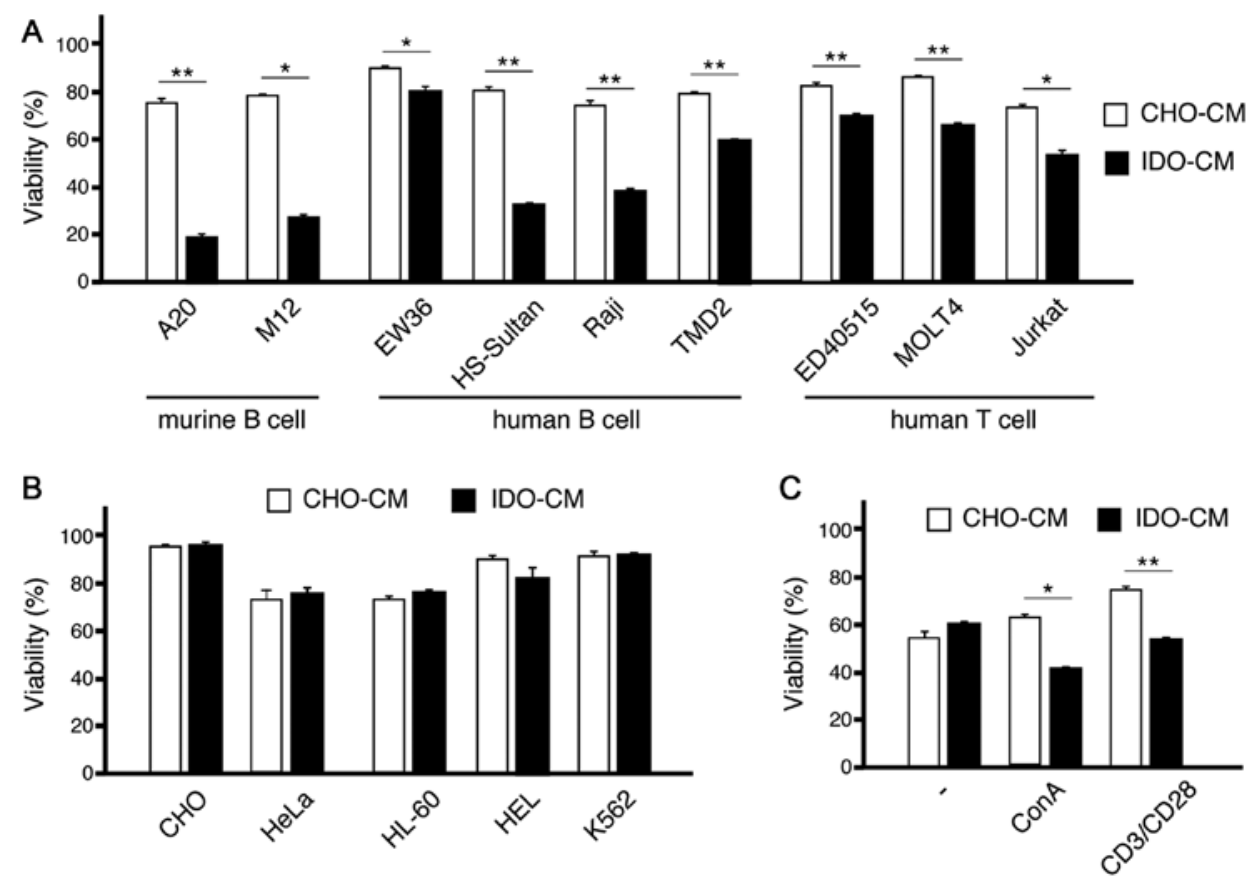

Figure 2. Effect of conditioned medium with CHO-IDO (IDO-CM) on murine and human lymphoid cell lines. (A) Murine B-cell lines, human B-cell or T-cell lines, and (B) non-lymphoid cell lines were cultured in CHO-CM (white bar) or IDO-CM (black bar) for 2 days. The viabilities were determined by flow cytometry. Results are presented as means \pm SD of triplicate cultures. The astersks indicate statistically significant differences determined by Student's t-test $\left({ }^{*} \mathrm{P}<0.01,{ }^{* *} \mathrm{P}<0.001\right)$. (C) PBMCs from healthy donors were cultured with $30 \mathrm{U} / \mathrm{ml} \mathrm{IL}-2$. The viabilities of cells were analyzed after 2 days of culture in CHO-CM or IDO-CM added to $10 \mathrm{ng} / \mathrm{ml}$ of concavalin A (ConA) or human T-activated CD3/CD28 Dynabeads ("P $\left.<0.01,{ }^{* *} \mathrm{P}<0.001\right)$.
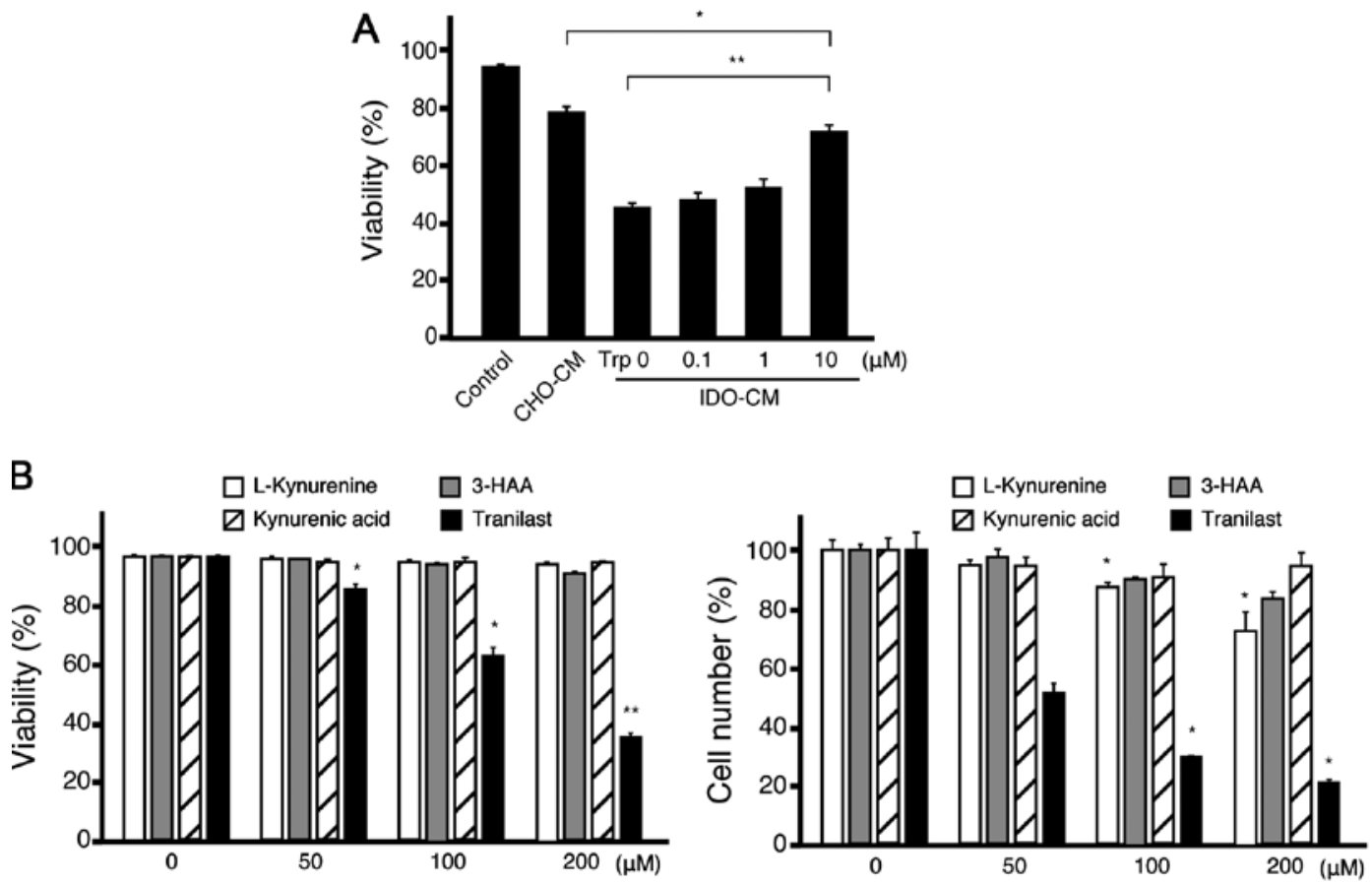

Figure 3. Effects of exogenous tryptophan or various tryptophan metabolites on viabilities of murine B cell line. (A) The viabilities of A20 were analyzed after culture with added IDO-CM and excess tryptophan $(0,0.1,1$ and $10 \mu \mathrm{M})$. The astersks indicate statistically significant differences $\left({ }^{*} \mathrm{P}<0.01,{ }^{* *} \mathrm{P}<0.001\right)$. (B) The viabilities (right) and viable cell numbers (left) of A20 are shown after 2 days of culture with various tryptophan metabolites (L-Kynurenine, 3-HAA, Kynurenic acid, tranilast) at the indicated concentrations. The astersks indicate significant differences compared with untreated cells $\left({ }^{*} \mathrm{P}<0.01,{ }^{* *} \mathrm{P}<0.001\right)$.

cells, but not with the CLL-B cells after the separation of B cells and non-B cells (Fig. 1C).
Establishment of cell lines transfected with the murine IDO gene. Next, we cloned the murine IDO cDNA from the spleno- 

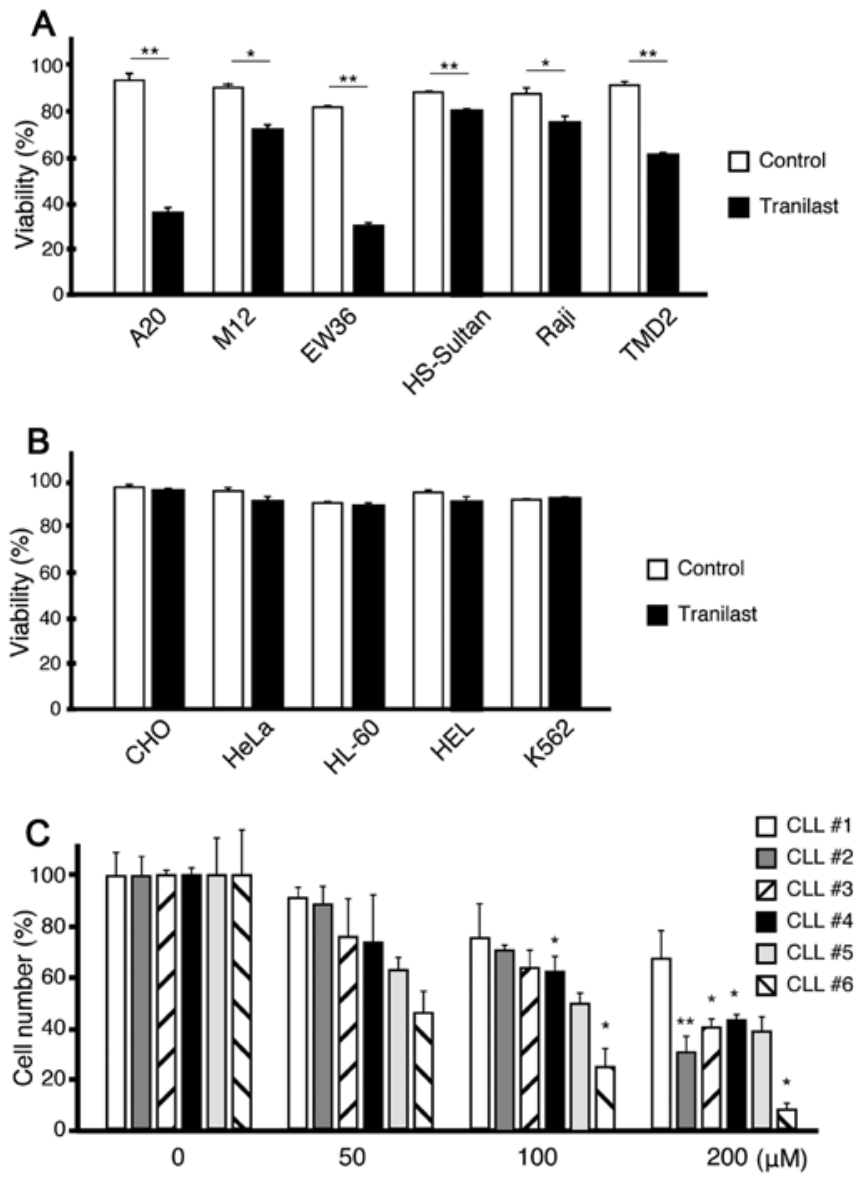

Figure 4. Effects of tranilast on the viability of cells. Lymphoid cell lines (A) or myeloid or epithelial cell lines (B) were cultured in a medium treated with or without $200 \mu \mathrm{M}$ of tranilast for $48 \mathrm{~h}$. Mean viability of the triplicate cultures is shown. The astersks indicate significant differences in compared with untreated cells ( $\left.{ }^{*} \mathrm{P}<0.01,{ }^{* *} \mathrm{P}<0.001\right)$. (C) PBMCs from 6 patients with CLL were treated with indicated concentrations of tranilast. The viabilities of cells were analyzed after 4 days of culture, and mean values from triplicates are shown. The fraction of CLL B cells determined with flow cytometry was $>90 \%$ of every PBMC. The cell number (\%) was expressed as a percentage relative to the untreated control cells. The astersks indicate significant differences in compared with untreated cells $\left({ }^{*} \mathrm{P}<0.01,{ }^{* *} \mathrm{P}<0.001\right)$.

cytes of the $\mathrm{BALB} / \mathrm{c}$ mice in the pcDNA3 vector containing the G418-resistant gene, and transfected it into CHO cells. Multiple clones were selected by G418 that had high cytoplasmic IDO protein as well as IDO mRNA expression confirmed by immunoblot and RT-PCR (Fig. 1D, CHO-IDO). We next introduced the $I D O$ gene into the murine B-cell lines A20 and M12 to examine the effect of IDO on the development of lymphoid malignancies. Compared with the control vector without the insert, the $I D O$-containing vector produced an almost equal number of G418-resistant clones. Although comparable amounts of IDO mRNA to CHO-IDO could be detected in these cells by RT-PCR, IDO protein was not detected, which suggested post-transcriptional downregulation of IDO in these lymphoid cell lines (Fig. 1D). Although some proteins have been regulated by their stability post-translationally, the proteasome inhibitors could not induce the IDO expression in these transfectants (data not shown).

Conditioned medium with CHO-IDO is cytotoxic to lymphoid cell lines. This downmodulation allowed us to consider the possibility that IDO may be toxic to these lymphoid cell lines. To examine this possibility, we created the conditioned medium with $\mathrm{CHO}$ (CHO-CM) and CHO-IDO (IDO-CM), and cultured these murine lymphoid cell lines in this media. The viability of both A20 and M12 was markedly decreased in IDO-CM compared with those in CHO-CM (Fig. 2A).

We then examined the viability of various cell lines cultured in CHO-CM or IDO-CM. All lymphoid cell lines examined, including both T-cell and B-cell lines, showed less viability in IDO-CM than in CHO-CM (Fig. 2A). In contrast, the viability of non-lymphoid cell lines in IDO-CM was not changed significantly in comparison with those in CHO-CM (Fig. 2B).

When T cells were cultured with only $30 \mathrm{U} / \mathrm{ml} \mathrm{IL}-2$, these cells showed similar viability in IDO-CM than in CHO-CM. However, when $\mathrm{T}$ cells were cultured with $10 \mathrm{ng} / \mathrm{ml}$ concanavalin A (ConA) or human T-activated CD3/CD28 Dynabeads, IDO-CM decreased their viability (Fig. 2C) indicating that signals from antigenic stimuli may induce susceptibility to IDO toxicity.

Collectively, the cytotoxic effect of IDO may be specific to activated lymphocytes and lymphoid lines.

Tryptophan metabolites inhibit the proliferation of lymphoid cell lines. Both the starvation of tryptophan and the production of tryptophan metabolites induced by IDO have been reported to be responsible for IDO immune suppression (2). Actually the concentration of trypatophan in IDO-CM was undetectable (data not shown). Therefore, we cultured IDO-sensitive lymphoid lines with added IDO-CM and an excess amount of tryptophan. The addition of tryptophan restored the viability in IDO-CM, though the restoration was not complete (Fig. 3A); this indicated that tryptophan metabolites as well as the starvation of tryptophan affected the lymphoid lines.

We then examined the effects of the various tryptophan metabolites on the viability of IDO-sensitive lymphoid line A20 (Fig. 3B). After treatment with L-Kynurenine or 3-HAA, the viable number of A20 cells was decreased although the viability had not changed. These results indicated that these molecules could suppress proliferation although they were not cytotoxic. Kynurenic acid could not change proliferation. Compared with these molecules, tranilast markedly decreased the viability of A20 cells. Tranilast not only induced cell death, but also attenuated the proliferation of A20 cells, as estimated by carboxyfluorescein succinimidyl ester (CFSE) dilution assay (data not shown). This effect appears to be independent of the cell cycle phase because the treated cells remained unchanged by the cell cycle analysis (data not shown). When tranilast was washed out from the medium after 1 day of treatment, A20 cells proliferated as much as the untreated cells, indicating that the effect of tranilast was reversible at this time-point. However, the A20 cells did not proliferate after 2 days of tranilast treatment (data not shown). We then examined the effect of tranilast in combination with chemotherapeutic reagents. Tranilast showed additive cytotoxicity with active metabolites of 4-hydroperoxycyclophosphamide and fludarabine (data not shown).

Tranilast induces cell death of lymphoid malignant cells. We examined the effect of tranilast treatment on various cell 
A

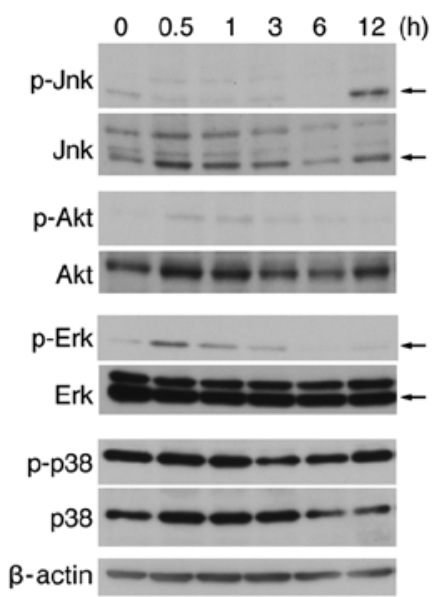

B
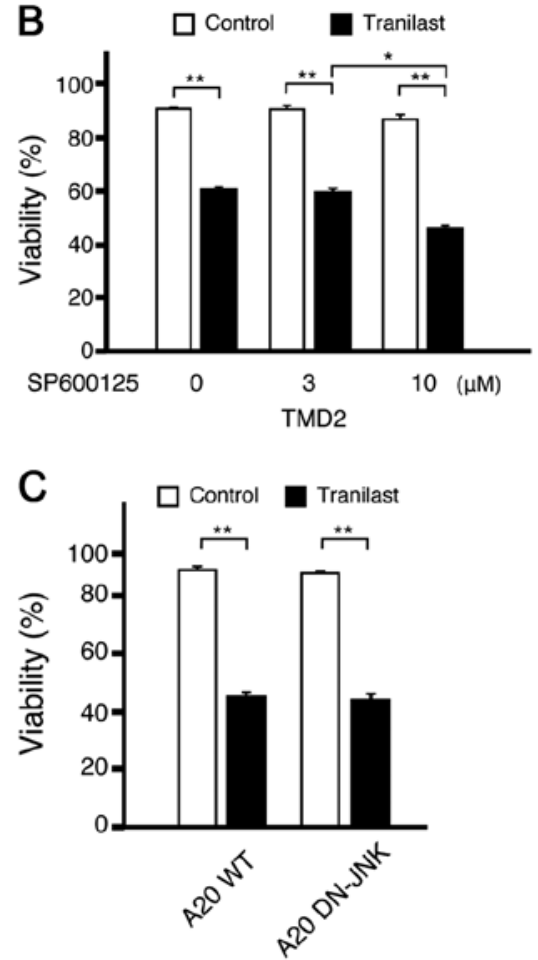

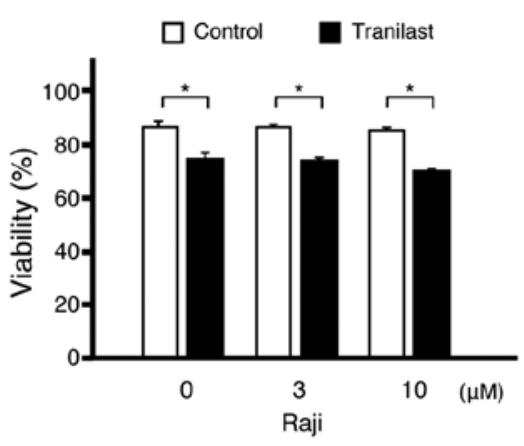

Figure 5. c-Jun N-terminal kinase (JNK) activation induced by tranilast. (A) A20 cells were treated with $200 \mu \mathrm{M}$ of tranilast and harvested at the indicated time. The whole cell lysate was subjected to immunoblot using antibodies against the indicated anti-phosphorylated proteins (p-JNK, p-Akt, p-Erk and p-p38). The same membranes were reprobed with anti-total proteins (JNK, Akt, Erk and p38). The major band of p-JNK was the $46 \mathrm{kD}$ form of JNK, and the major band of p-Erk was the $42 \mathrm{kD}$ form of Erk (Erk-2) as determined by the comparison with bands of total JNK or Erk (arrows). (B) The human B cell lines, TMD2 and Raji, were treated with or without $200 \mu \mathrm{M}$ of tranilast in addition to the indicated concentration of the JNK inhibitor, SP600125. After 2 days of culture, the viabilities were analyzed and shown as the means with SD of triplicates. (C) A20 was transfected with pQCXIN-dominant negative form of JNK2 and selected with G418. Enhanced $54 \mathrm{kD}$ of JNK and diminished auto-phosphorylation of $46 \mathrm{kD}$ of JNK were verified by immunoblot analysis (data not shown). The viabilities were analyzed after 2 days of culture with $200 \mu \mathrm{M}$ of tranilast. The astersks indicate significant differences in compared with untreated cells $\left({ }^{*} \mathrm{P}<0.01,{ }^{* *} \mathrm{P}<0.001\right)$.

lines. Similar to IDO-CM, tranilast induced cell death in all lymphoid lines (Fig. 4A) but not in the myeloid or epithelial cell lines (Fig. 4B) examined; however, the effects of IDO-CM and tranilast were not totally equivalent. Tranilast induced more cell death of EW36 than IDO-CM, and conversely, IDO-CM was more toxic than tranilast for HS-Sultan. We also analyzed the effect of tranilast against the primary samples from six patients with CLL. Tranilast dose-dependently augmented the spontaneous apoptosis of CLL cells (Fig. 4C).

Tranilast induces the phosphorylation of JNK. To reveal the molecular mechanism of cytotoxicity induced by tranilast, we checked the activation of some kinases in A20, TMD2 (15), and Raji cells by immunoblot after tranilast treatment. The phosphorylation of $42 \mathrm{kD}$ of extracellular signal-regulated kinase (Erk-2) was upregulated transiently after the addition of tranilast in these lines (Fig. 5A; and data not shown). In addition, JNK activation was induced in A20 cells $12 \mathrm{~h}$ after tranilast treatment (Fig. 5A). Although JNK activation was not obvious in the human B cell lines, TMD2 and Raji, after the addition of tranilast alone, tranilast clearly augmented the phosphorylation of JNK after B-cell receptor signaling using anti-IgM treatment (data not shown). Because the JNK activation could promote not only cell survival but also cell death depending on the cell type, we examined the effect of JNK inhibition on tranilast treatment. The addition of JNK inhibitor SP600125 augmented the cytotoxicity of tranilast only slightly (Fig. 5B). We also induced the dominant negative form of JNK2 into some A20 cells. Tranilast induced the cell death of these cells as compared to that of wild-type A20 cells, indicating that JNK was not required for the cytotoxicity of tranilast (Fig. 5C).

The effect of tranilast is independent of aryl hydrocarbon receptor $(A h R)$. Kynurenines can act as endogenous AhR ligand $(16,17)$. Furthermore, tranilast could act as a ligand for AhR (18). To examine if the cytotoxic effect of tranilast on lymphoid cells was mediated by AhR, the AhR antagonist, $\alpha$-naphthoflavone ( $\alpha$-NF), was added to the culture with tranilast. The addition of $\alpha$-NF did not change the effect of tranilast (Fig. 6A). In addition, the potent agonist of AhR, 2,3,7,8-tetrachlorodibenzodioxin (TCDD), was not as cytotoxic to A20 cells as tranilast (Fig. 6B). Collectively, the cytotoxic effect of tranilast appeared to be independent of AhR.

Tranilast attenuates the growth of lymphoid tumor in vivo. To examine the effect of tranilast in vivo, we inoculated A20 into BALB/c mice subcutaneously. After 7 days of inoculation, treatment with oral administration of $100 \mathrm{mg} / \mathrm{kg}$ of tranilast suspended in CMC solution or CMC solution alone was started. The treatment was applied twice daily for 3 weeks. The mice that underwent tranilast treatment had 

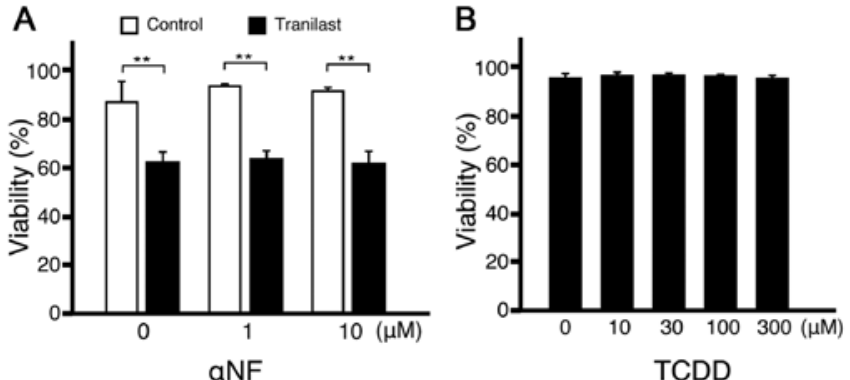

Figure 6. Cytotoxic effect of tranilast on lymphoid cells was independent of the aryl hydrocarbon receptor (AhR). (A) A20 cells were cultured with or without $200 \mu \mathrm{M}$ tranilast in addition to $\alpha$-naphthoflavone $(\alpha-\mathrm{NF})$, which is an $\mathrm{AhR}$ antagonist at the indicated concentration. The viability after 2 days of culture is indicated. The astersks indicate significant differences compared with untreated cells $\left({ }^{* *} \mathrm{P}<0.001\right)$. (B) The viability of A20 cells cultured in a medium treated with AhR agonist, 2,3,7,8-tertrachlorodibenzodioxin (TCDD), at the indicated concentration for 2 days are shown.

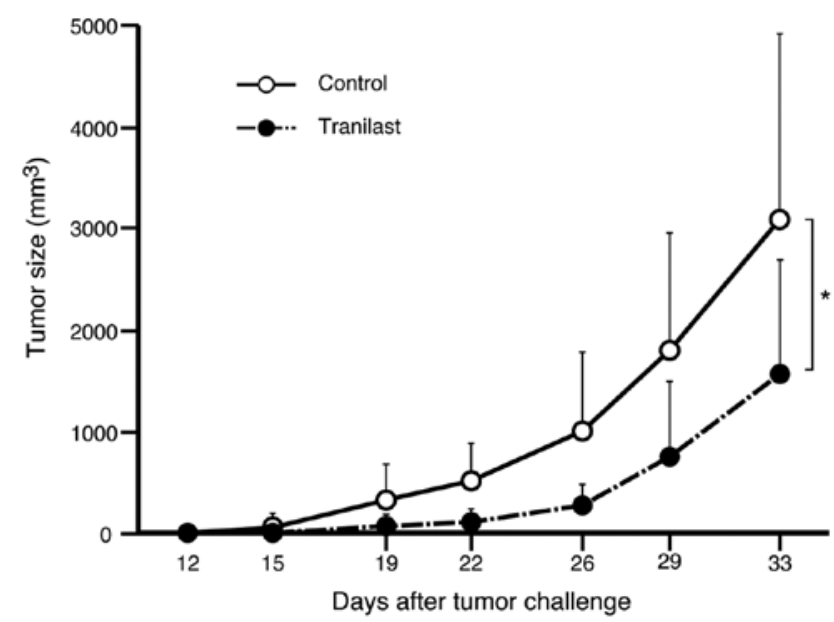

Figure 7. In vivo efficacy of tranilast in a mouse lymphoma model. Six $\mathrm{BALB} / \mathrm{c}$ mice were engrafted with A20 cells subcutaneously and treated with $100 \mathrm{mg} / \mathrm{kg}$ of tranilast suspended in 5\% carboxymethyl cellulose (CMC) solution by oral administration. Mice dosed with CMC solution alone $(n=7)$ were used as controls. Mean tumor volumes of mice are plotted. The difference at day 33 was significant $\left({ }^{*} \mathrm{P}=0.02\right)$.

slower tumor development compared with the mice that were dosed with CMC solution alone. The tumor mass at 5 weeks after the challenge was significantly smaller in mice treated with tranilast compared with the control mice $(\mathrm{P}=0.02$, Fig. 7).

\section{Discussion}

In the present study, we showed that conditioned medium with IDO-expressing cells and a tryptophan metabolite, tranilast, both decreased proliferation and induced cell death of activated lymphocytes and specifically lymphoid tumor cells. In agreement with our results, it has been reported that mesenchymal stem cells treated with IFN $\gamma$ inhibited the proliferation of normal and follicular lymphoma B cells in an IDO-dependent manner (19). Because tryptophan is an essential amino acid, its starvation and the presence of metabolites could influence all tissues including the placenta (4) or dendritic cells (6), which contain a significant amount of IDO. The specificity of cytotoxicity induced by IDO against activated lymphoid cells may explain the machinery for the protection of IDO-expressing cells themselves. In agreement with this result, we could not get an increased exogenous expression of IDO protein in murine lymphoid cell lines; however, we detected $I D O$ mRNA in these transfected cells. In the same way, it has been reported that IDO protein has weak or absent enzymatic activity in human B cells, although it can be induced by CD40L and IFN $\gamma$ together or by a TLR agonist (20). We analyzed IDO expression in lymphoid tumor samples by RT-PCR (Fig. 1A). The expression in tumors was not increased compared with those in PBMCs from healthy donors. Conversely, the $I D O$ expression in most tumor samples were weak and in some barely detectable, particularly in BL. Because the percentage of malignant B cells in BL were almost $100 \%$ (data not shown), the decrease of $I D O$ in malignant tissues may represent the decrease of IDO-expressing non-B cells. In agreement of this, the IDO expression was mainly derived from non-malignant bystander cells from the samples examined. A report has described that the functional expression of IDO could be detected in a portion of diffuse large B-cell lymphoma (DLBCL) cases, and this expression was correlated with worse prognosis (21). Although we did not have any IDO-expressing DLBCL cases, these IDO-expressing lymphoma cells may have the same protective properties as non-lymphoid cells against IDO toxicity. It was also possible that this property could result in resistance to chemotherapeutic reagents and contribute to the poor prognosis and escape from immunological surveillance.

As tranilast was the most effective tryptophan metabolite for EAE as reported by Platten et al (10), it was also the most potent cytotoxic reagent for lymphoid tumor cells among the tryptophan metabolites examined. Tranilast has an inhibitory effect on various cancer cells including breast, prostate cancer and glioma (22). In our analysis, lymphoid cell lines were more susceptible to the cytotoxicity of tranilast than the non-lymphoid cells examined. As kynurenines can act as endogenous AhR agonists and suppress immune response, tranilast is also an AhR agonist. Its effect on breast cancer stem cells is AhR dependent (18). The effect of tranilast for lymphoid tumor was not inhibited by AhR antagonist $\alpha-\mathrm{NF}$ indicating that the effect was independent of AhR in the present study. To date, the mechanisms of specific cytotoxicity to lymphoid tumor cells induced by tranilast have not been elucidated. Tranilast induced Erk-2 activation transiently and JNK activation after 1 day of treatment in A20 cells. In contrast, it was reported that tranilast suppressed LPS-induced Erk-2 activation in microglial cells (23) and IL-1 $\beta$-induced JNK activation of mesangial cells (24). These opposite effects of tranilast on MAP kinase signaling may explain the specific suppression against lymphoid cells. JNK can mediate cell survival and cell death dependent on cell type $(25,26)$. But the fact that tranilast could not be inhibited by the inhibitor or the dominant negative form of JNK indicated that JNK was not essential for the effect of tranilast. Because JNK was activated by cellular stress such as UV, tranilast may also induce similar stress in lymphoid malignant cells.

Tranilast has been widely used as oral anti-allergic reagent in Japan and South Korea, and its safety is well recognized. 
Because infectious complications have not been reported as the side-effect of tranilast, this reagent should not induce profound immunosuppression. Adjuvant use of tranilast that potentiates the effect of chemotherapy is warranted.

\section{Acknowledgements}

The authors would like to thank Ms. Kaori Okada, Ms. Sakie Endo, Ms. Ayumi Kiyokawa and Ms. Shihomi Endo for skillful technical assistance and Dr Toshie Suzuki and Dr Hideki Kudo for helpful discussions. The authors also thank Kissei Pharmaceutical Corporation for providing the tranilast, and Dr Roger Davis for providing the Jnk2a2 plasmid.

\section{References}

1. Fallarino F, Grohmann U, Vacca C, et al: $\mathrm{T}$ cell apoptosis by tryptophan catabolism. Cell Death Differ 9: 1069-1077, 2002.

2. Fallarino F, Grohmann U, You S, et al: The combined effects of tryptophan starvation and tryptophan catabolites down-regulate $\mathrm{T}$ cell receptor zeta-chain and induce a regulatory phenotype in naive T cells. J Immunol 176: 6752-6761, 2006.

3. Frumento G, Rotondo R, Tonetti M, Damonte G, Benatti U and Ferrara GB: Tryptophan-derived catabolites are responsible for inhibition of $\mathrm{T}$ and natural killer cell proliferation induced by indoleamine 2,3-dioxygenase. J Exp Med 196: 459-468, 2002.

4. Munn DH, Zhou M, Attwood JT, et al: Prevention of allogeneic fetal rejection by tryptophan catabolism. Science 281: 1191-1193, 1998.

5. Mellor AL, Sivakumar J, Chandler P, et al: Prevention of T celldriven complement activation and inflammation by tryptophan catabolism during pregnancy. Nat Immunol 2: 64-68, 2001.

6. Mellor AL and Munn DH: IDO expression by dendritic cells: tolerance and tryptophan catabolism. Nat Rev Immunol 4 $762-774,2004$

7. Inaba T, Ino K, Kajiyama H, et al: Role of the immunosuppressive enzyme indoleamine 2,3-dioxygenase in the progression of ovarian carcinoma. Gynecol Oncol 115: 185-192, 2009.

8. Ino K, Yamamoto E, Shibata K, et al: Inverse correlation between tumoral indoleamine 2,3-dioxygenase expression and tumor-infiltrating lymphocytes in endometrial cancer: its association with disease progression and survival. Clin Cancer Res 14: 2310-2317, 2008

9. Sharma MD, Baban B, Chandler P, et al: Plasmacytoid dendritic cells from mouse tumor-draining lymph nodes directly activate mature Tregs via indoleamine 2,3-dioxygenase. J Clin Invest 117: 2570-2582, 2007.

10. Platten M, Ho PP, Youssef S, et al: Treatment of autoimmune neuroinflammation with a synthetic tryptophan metabolite. Science 310: 850-855, 2005.

11. Jasperson LK, Bucher C,Panoskaltsis-Mortari A, et al: Indoleamine 2,3-dioxygenase is a critical regulator of acute graft-versus-host disease lethality. Blood 111: 3257-3265, 2008.
12. Jasperson LK, Bucher C, Panoskaltsis-Mortari A, Mellor AL, Munn DH and Blazar BR: Inducing the tryptophan catabolic pathway, indoleamine 2,3-dioxygenase (IDO), for suppression of graft-versus-host disease (GVHD) lethality. Blood 114: 5062-5070, 2009.

13. Gupta S, Barrett T, Whitmarsh AJ, et al: Selective interaction of JNK protein kinase isoforms with transcription factors. EMBO J 15: 2760-2770, 1996.

14. Hu D and Kipps T: Reduction in mitochondrial membrane potential is an early event in Fas-independent CTL-mediated apoptosis. Cell Immunol 195: 43-52, 1999.

15. Tohda S, Nara N, Murohashi I and Aoki N: Establishment of an interleukin-3-dependent leukemic cell line from a patient with chronic lymphocytic leukemia in the acute phase. Blood 78: 1789-1794, 1991.

16. Opitz CA, Litzenburger UM, Sahm F, et al: An endogenous tumour-promoting ligand of the human aryl hydrocarbon receptor. Nature 478: 197-203, 2011.

17. Nguyen NT, Kimura A, Nakahama T, et al: Aryl hydrocarbon receptor negatively regulates dendritic cell immunogenicity via a kynurenine-dependent mechanism. Proc Natl Acad Sci USA 107: 19961-19966, 2010.

18. Prud'homme GJ, Glinka Y, Toulina A, Ace O, Subramaniam V and Jothy S: Breast cancer stem-like cells are inhibited by a non-toxic aryl hydrocarbon receptor agonist. PLoS One 5: e13831, 2010

19. Maby-El Hajjami H, Amé-Thomas P, Pangault C, et al: Functional alteration of the lymphoma stromal cell niche by the cytokine context: role of indoleamine-2,3 dioxygenase. Cancer Res 69: 3228-3237, 2009.

20. Godin-Ethier J, Hanafi LA, Duvignaud JB, Leclerc D and Lapointe R: IDO expression by human B lymphocytes in response to T lymphocyte stimuli and TLR engagement is biologically inactive. Mol Immunol 49: 253-259, 2011.

21. Ninomiya S, Hara T, Tsurumi H, et al: Indoleamine 2,3-dioxygenase in tumor tissue indicates prognosis in patients with diffuse large B-cell lymphoma treated with R-CHOP. Ann Hematol 90: 409-416, 2011.

22. Rogosnitzky M, Danks R and Kardash E: Therapeutic potential of tranilast, an anti-allergy drug, in proliferative disorders. Anticancer Res 32: 2471-2478, 2012.

23. Platten M, Eitel K, Wischhusen J, Dichgans J and Weller M: Involvement of protein kinase Cdelta and extracellular signalregulated kinase-2 in the suppression of microglial inducible nitric oxide synthase expression by N-[3,4-dimethoxycinnamoyl]anthranilic acid (tranilast). Biochem Pharmacol 66: 1263-1270, 2003.

24. Chikaraishi A, Hirahashi J, Takase O, et al: Tranilast inhibits interleukin-1beta-induced monocyte chemoattractant protein-1 expression in rat mesangial cells. Eur J Pharmacol 427: 151-158, 2001.

25. Johnson GL and Nakamura K: The c-jun kinase/stress-activated pathway: regulation, function and role in human disease. Biochim Biophys Acta 1773: 1341-1348, 2007.

26. Behrens A, Sibilia M and Wagner EF: Amino-terminal phosphorylation of c-Jun regulates stress-induced apoptosis and cellular proliferation. Nat Genet 21: 326-329, 1999. 